\title{
Hemispheric differences in semantic processing: Category matching is not the same as category membership
}

\author{
PETER J. URCUIOLI, RAYMOND M. KLEIN, and JAMES DAY \\ Dalhousie University, Halifax, Nova Scotia B3H 4JI, Canada
}

\begin{abstract}
Two closely related semantic processing tasks were studied under identical procedural conditions in order to examine lateral visual field effects on reaction times. In Experiment 1, reaction times did not differ as a function of visual field when subjects decided whether a lateral word was a member of a foveally presented category word (category membership task). On the other hand, reaction times were faster for right than for left visual field stimulus presentations when subjects decided whether two words, one lateral and one foveal, belonged to the same category (category matching task), although this advantage did not occur immediately. In Experiment 2, the laterality effect in the category matching task was studied as a function of word familiarity. Two groups of subjects performed the matching task for two blocks of trials, one group receiving the same word list in each block and the other receiving different lists. No visual field differences in reaction times were observed for either group during the first block of trials, but a distinct right field advantage appeared for both during the second block. The data from these experiments suggest that category matching strategies rely upon structures or processes localized in the left hemisphere, although their influence is not immediate. Category membership strategies, on the other hand, do not depend upon such localized structures.
\end{abstract}

Converging evidence from several domains has led to the conclusion that the human cerebral hemispheres differ from one another in their functional characteristics. Most notably, the capacity for language production in most right-handed individuals is localized in the left hemisphere (Gazzaniga, 1970). It is also generally agreed that, for right-handers, the left hemisphere is superior to the right in other sorts of linguistic processing. Nonetheless, in research with splitbrain patients and other clinical populations, the right hemisphere has been shown to have a significant functional receptive vocabulary (Hecaen, 1978; Levy \& Trevarthen, 1977; Searleman, 1977; Zaidel, 1978). This raises two important questions. First, to what extent can the clinical findings be generalized to the right hemisphere of normal right-handers whose cerebral hemispheres have not been disconnected or otherwise damaged? Second, what are the differences between the normal right and left hemispheres in their control of language-related functions?

Recent studies (e.g., Day, 1977; Hardyck, Tzeng, \& Wang, 1978; McFarland, McFarland, Bain, \& Ashton, 1978) have begun to take aim at these questions. In

This research was supported by grants from the National Research Council of Canada and the Dalhousie University Development Fund. The authors would like to thank Marilyn Klein for technical assistance, Andreina Brady and Florence Fertig for typing the manuscript, and John Barresi for his comments on an earlier draft. Requests for reprints should be addressed to Peter J. Urcuioli, who is now at Sensory Sciences Center, P.O. Box 20708, Room 7.242, University of Texas, Houston, Texas 77025. these studies, verbal material is presented to the left and right sensory half-fields, and the accuracy and/or speed of corresponding discriminative responses is then measured. Performance for each field condition reflects in part the functioning of the contralateral cerebral hemisphere. Consequently, differences in performance are an index of hemispheric asymmetry in language-related processing-assuming, of course, that there are no peripheral sensory deficits. Conversely, equivalent halffield performance indicates that both hemispheres have the capacity to perform the required task.

Day (1977), for example, found that concrete words and adjectives are recognized equally rapidly by normal right-handers in either the left or right visual fields (LVF and RVF, respectively). Recognition of verbs and abstract nouns, on the other hand, was faster in the RVF than in the LVF. In Day's experiment, subjects had to decide as quickly as possible whether or not a visually presented letter string spelled a word (lexical decision task). His data suggest that the normal right hemisphere can process certain linguistic elements as efficiently as the left, although it is apparently inferior for other elements.

In a companion experiment, Day (1977, Experiment 3 ) also found that the right hemisphere could detect the semantic relationship between certain nouns and their superordinate categories as efficiently as could the left hemisphere. On each trial of this experiment, one of the six category words (three concrete, three abstract) was presented to subjects at fixation, and a positive or negative instance of the cate- 
gory appeared simultaneously in either the LVF or RVF. Subjects responded only if the lateral word was a member of the foveally presented category word. Once again, Day found no visual field reaction time (RT) differences when the stimuli for this task were concrete words and their superordinate categories.

Apparently contradictory results can be seen, however, in a previous experiment by Gross (1972, Experiment 1). In her experiment, pairs of concrete words were presented simultaneously to subjects, although both words appeared laterally. Subjects responded in one of two ways, depending on whether the words belonged to the same or different categories. Gross found that RTs for both "same" and "different" responses were faster when stimuli appeared in the RVF, effects that were independent of response mode (manual vs. vocal).

The difference between Day's (1977) results and those reported by Gross (1972) is surprising in view of the similarity between their stimuli and task requirements. Since the existence of this discrepancy precludes firm conclusions about the nature of the right hemisphere's capacity to detect semantic relationships, it behooves us to determine its basis. Three possible explanations are considered below.

First, and perhaps most likely, one of the many procedural differences between the two studies (e.g., manner of stimulus presentation and response requirements) might account for their conflicting results. This explanation was favored by Day (1977, p. 525), who emphasized that, in Gross's manualresponse condition, either hemisphere could control performance. In this situation, the left hemisphere may have taken control regardless of the field of presentation by virtue of its language dominance. Such a strategy would produce a RVF-left hemisphere advantage, even though both hemispheres were equally capable of performing the task.

Second, and most interestingly, it is possible that subtle differences in task requirements may underlie the different results. Day's subjects determined if a word was a member of a particular category (category membership), while Gross's subjects determined if two words were from the same category (category matching). Perhaps the latter discrimination requires neural structures or processes localized in the left hemisphere, while the former can rely upon circuitry in either hemisphere.

Finally, it is possible that one or both of these findings is spurious.

The purpose of the first experiment, therefore, was to determine if the conflicting results obtained by Gross and Day were caused by procedural differences or by differences in task requirements. To distinguish between these alternatives, we had subjects perform the two tasks using the same words and procedures.

\section{EXPERIMENT 1}

\section{Method}

Subjects. The 20 subjects ( 14 males, 6 females) who participated in this experiment were selected from the staff and student populations of the departments of Geology, Oceanography, and Psychology at Dalhousie University. All were right-handed, with laterality quotients on the Edinburgh Inventory (Oldfield, 1971) ranging from .75-1.0. No subject reported any incidence of familial sinistrality among parents or siblings. Furthermore, their inclusion in the experiment was dependent upon a high degree of performance accuracy, such that their error rate under each visual field condition did not exceed $25 \%$. This assured a relatively large sampling of RTs from each visual field. The subjects were divided equally into two groups (see Procedure); mean laterality quotients for these groups were .92 (Group D-G) and .86 (Group G-D).

Stimuli. The verbal stimuli for this experiment consisted of eight three-letter words denoting animals (cat, cow, dog, elk, hen, pig, rat, ape), eight three-letter words denoting parts of the human body (ear, eye, hip, jaw, leg, rib, toe, arm), and the two category words "animal" and "body." The three-letter words were taken from the list reported by Gross (1972). Two sets of 64 stimulus configurations were prepared, one set for a replication of Day's category membership task (Task D) and the remaining set for a replication of Gross's category matching task (Task G).

Each stimulus configuration for Task D consisted of two words oriented vertically, one appearing at the center of fixation and one appearing laterally. The foveal word was either the category word "animal" or the category word "body." The lateral word was drawn from the list of 16 three-letter words. Sixteen "same" units were constructed by pairing each three-letter word with its appropriate category word. Similarly, 16 "different" units were constructed by pairing each three-letter word with the opposite category word. Two configurations were prepared for each "same" and "different" unit: one with the lateral word to the left of the category word and one with the lateral word to the right of the category word.

The stimulus configurations for Task $G$ also consisted of two vertically oriented words, one foveal and one lateral. "Same" units were prepared by selecting 16 configurations from all possible configurations $(8 \times 7=56)$ consisting of pairs of three-letter words belonging to the same category (i.e., both animals or both parts of the human body). Sixteen "different" units, consisting of pairs of three-letter words belonging to different categories (i.e., one animal and one body part), were selected in a similar fashion. The only constraint upon selection of these units was that the frequency of occurrence of each lateral word match the corresponding frequency for that same word in Task D. For each of the resulting 32 stimulus configurations, the latter word appeared once to the left and once to the right of the foveal word.

Pairs of words were typed on high-reflectance Chrome-Kote paper with a 10-pitch lowercase Orator typeface. The stimulus pairs were then photographed and mounted in $35-\mathrm{mm}$ glass slides, appearing black on white backgrounds. When viewed at a distance of $58 \mathrm{~cm}$, the three-letter words subtended visual angles of $7.8 \mathrm{deg}$ vertical and $1.5 \mathrm{deg}$ horizontal. The four-letter word "body" and the six-letter word "animal" subtended angles of 8.8 and $16.3 \mathrm{deg}$ vertical, respectively, and $1.5 \mathrm{deg}$ horizontal. The distance between the point of fixation (center of the foveal word) and the inner edge of the lateral word subtended a visual angle of $7.6 \mathrm{deg}$.

Apparatus. A slide projector (Kodak Carousel, Model B-2), with an electronic shutter (Ralph Gerbrands, Model 66) mounted on the lens, projected the stimuli onto a $49 \times 59.7 \mathrm{~cm}$ screen located $83.8 \mathrm{~cm}$ in front of the projector. A digital timer control unit (Ralph Gerbrands, Model 300) set at $150 \mathrm{msec}$ provided a constant stimulus duration. A neutral density 1.0 filter was attached to the shutter aperture in order to decrease stimulus intensity to a level that eliminated afterimages. The response manipulandum was a 
small microswitch housed in a metal box positioned directly in front of the subject. The rates of stimulus presentation and reaction times (RTs) were monitored by a Linc- 8 computer located in a separate room.

Procedure. The subjects sat at one end of an $87 \times 183 \mathrm{~cm}$ table, facing the projection screen, and positioned their heads in a chin- and headrest during the experiment in order to maintain a constant viewing distance $(58 \mathrm{~cm})$.

Prior to each task, the subjects read instructions detailing the nature of the task and the two categories of words they would see. They were told that two words, oriented vertically and appearing simultaneously, would flash briefly on the projection screen on each trial. For Task D, the subjects were told to press the response button as quickly as possible if the word presented laterally was an instance of the foveally presented category word ("animal" or "body"); otherwise, they were not to respond. For Task G, the subjects were told to press the button if both the lateral word and the foveal word belonged to the same category (i.e., both animals or both parts of the human body), but not to press it otherwise.

Each task consisted of 16 practice and 64 test trials. A separate list of four-letter animal and body words, typed and photographed in the same manner as the three-letter words, was used for the practice trials. Test trials were split into two 32-trial blocks, each containing equal numbers of positive ("same") and negative ("different") trials. Within each 32-trial block, each lateral word appeared twice, once on a positive trial and once on a negative trial. Each stimulus configuration appeared once within a block, with the visual field location of the lateral word counterbalanced between blocks.

The subjects were tested on both tasks, half receiving Task D first (Group D-G) and half receiving Task G first (Group G-D). Half of the subjects within each group were instructed to respond with their left index finger and half with their right index finger. The subjects were told to maintain fixation on a small white dot located at the center of the projection screen (i.e., center of the area where the foveal word appeared) throughout the experiment. Two seconds were allowed for a response to occur on each trial. Successive trials were separated by a 2 -sec intertrial interval, which immediately followed a response or a 2 -sec interval without a response. Both RTs and errors were recorded.

Data analysis. Reaction times beyond \pm 2.0 standard deviations of the mean for each condition were discarded from data analysis, and new means were calculated on the basis of the remaining data. In general, the shortest and/or longest RT in each visual field condition was discarded from each subject's data. Exclusion of highly discrepant RTs was considered to be important, since they could adversely affect conclusions concerning the presence vs. absence of visual field differences or the degree and direction of such differences. The discarded data were not treated as errors.

For statistical evaluation of the RT and error data, post hoc contrasts across the sample means were calculated in the manner described by Scheffé (1953). The observed variance-ratios were compared, however, with tables of new critical variance-ratios, $\mathrm{F}[\mathrm{E} a] ; v_{1}, v_{2}$ (Rodger, 1975a). These were used in preference to traditional tabled $F$ values so that Type I error rate could be con- trolled on a per-decision basis (as opposed to an experiment-wise basis). In the experiments reported here, $\mathrm{E} \alpha=.05$. Conclusions regarding visual field differences, or lack thereof, were made by selecting and testing a set of $v_{1}$ mutually orthogonal contrasts and assigning appropriate values to contrasts that were rejected or accepted. Rodger (1975b, Equation 23) has shown that when statistical decisions have been made under these conditions, the relationships among the true (population) means implied by the decision set can be determined. The particular sets of contrasts chosen for decision making, therefore, were ones that led to meaningful conclusions and to high correlations between implied and sample means.

\section{Results and Discussion}

The mean RTs for correct responses as a function of task, visual field, and responding hand are shown for both groups in Table 1. Also shown are mean RTs averaged across responding hand, since this between-subjects variable had no consistent influence on performance.

Two prominent effects can be seen in the sample data. First, RTs were generally shorter on the category membership task (D) than on the category matching task $(G)$, independent of the order of task presentation. Second, visual field differences in RTs were affected by both the task and the order in which it was presented. For category matching, RTs were shorter on the average for RVF than for LVF presentations, but the difference was much greater when the task was performed second (Group D-G) than when it was performed first (Group G-D). For category membership, on the other hand, average RTs were shorter for LVF than for RVF presentations during initial performance (Group D-G), but the reverse was true during later performance (Group G-D).

The statistical analyses of each group's RT data showed the following task effects. For Group D-G, category membership RTs did not differ as a function of visual field $[F(3,27)=.19]$, and they did not differ from RVF RTs in the subsequent category matching task $[F(3,27)=.29]$. Category matching $R T s$ for LVF presentations, however, were longer than the other three $[F(3,27)=2.40]$. These decisions imply that the RTs for Group D-G were ordered as follows: $L V F_{D}=R V F_{D}=R V F_{G}<L V F_{G}$. In other words, there were no visual field differences for category membership (D) but a RVF advantage for category matching (G). For Group G-D, neither category

Table 1

Mean Reaction Times (in Milliseconds) for Correct Responses in Experiment 1

\begin{tabular}{|c|c|c|c|c|c|c|c|c|}
\hline \multirow[b]{3}{*}{ Hand } & \multicolumn{4}{|c|}{ Group D-G } & \multicolumn{4}{|c|}{ Group G-D } \\
\hline & \multicolumn{2}{|c|}{ Membership (D) } & \multicolumn{2}{|c|}{ Matching (G) } & \multicolumn{2}{|c|}{ Matching (G) } & \multicolumn{2}{|c|}{ Membership (D) } \\
\hline & LVF & RVF & LVF & RVF & LVF & RVF & LVF & RVF \\
\hline $\begin{array}{l}\text { Left } \\
\text { Right }\end{array}$ & $\begin{array}{l}905.0 \\
990.8\end{array}$ & $\begin{array}{l}991.0 \\
957.0\end{array}$ & $\begin{array}{r}980.0 \\
1114.2\end{array}$ & $\begin{array}{r}943.2 \\
1035.8\end{array}$ & $\begin{array}{l}1055.4 \\
1015.0\end{array}$ & $\begin{array}{r}1067.6 \\
952.0\end{array}$ & $\begin{array}{l}927.8 \\
915.1\end{array}$ & $\begin{array}{l}919.6 \\
847.0\end{array}$ \\
\hline Average & 947.9 & 974.2 & 1047.1 & 989.5 & 1035.2 & 1010.1 & 921.5 & 883.3 \\
\hline
\end{tabular}

Note-Data are shown by task, visual field, and responding hand for both groups. 
matching nor category membership RTs differed as a function of visual field [matching: $F(3,27)=.16$; membership: $F(3,27)=.38$ ], although the latter were shorter overall than the former $[F(3,27)=7.46]$. This implies the following $R T$ ordering: $\mathbf{L V F}_{\mathbf{D}}=\mathbf{R V F}_{\mathbf{D}}<$ $\mathrm{LVF}_{\mathrm{G}}=\mathrm{RVF}_{\mathrm{G}}$. In other words, there were no visual field differences in either task, although category membership (D) RTs were shorter overall than category matching $(\mathrm{G})$ RTs.

Our conclusions are buttressed by an analysis that simply breaks down the direction of visual field advantages shown by the subjects as they performed each task. For category membership, approximately the same numbers of subjects showed LVF and RVF advantages in both Group D-G $(L V F=6, R V F=4)$ and Group G-D $(L V F=4, R V F=6)$. For category matching, advantages were divided almost equally across subjects in Group G-D $(\mathrm{LVF}=4, \mathrm{RVF}=6)$, but clearly favored the RVF in Group D-G $(L V F=2$, $\mathrm{RVF}=8$ ).

One other effect worthy of note is the interaction between visual field differences and responding hand during initial-task performance. The effect was quite pronounced in Group D-G for category membership $[F(1,8)=7.42]$. For Group G-D, the RT data for category matching suggest a similar effect, although its relatively small size may just as well be attributed to sampling error $[F(1,8)=1.11]$. Nonetheless, it is interesting that the pattern of results was identical for both groups.

The error data from this experiment, shown in Table 2, were also analyzed for visual field effects. An analysis of errors is important for two reasons. First, faster RTs in one visual field as opposed to another could occur because subjects are biased to respond quickly to any stimulus (correct or incorrect) presented to that field. This would be reflected in a higher false-positive error rate in the visual field producing the faster response (speed-accuracy tradeoff). Second, a high proportion of false negatives (misses) for a particular visual field condition might suggest that RTs in that condition are artificially inflated, since subjects would be biased in an opposite direction. In fact, statistical analyses of false positives and false negatives gave no indication of either sort of bias. False positives did not differ by visual field for either group on either task [Group D-G: $F(3,27)=.24$ and .00 for membership and matching, respectively; Group G-D: $F(3,27)=1.12$ and .06 for matching and membership, respectively]. The same was true of false negatives [Group D-G: $F(3,27)=.02$ and .74 for membership and matching, respectively; Group G-D: $F(3,27)=.13$ and .82 , for matching and membership, respectively].

The results of this experiment thus replicate the findings of both Day (1977, Experiment 3) and Gross (1972, Experiment 1), suggesting that their differential effects were not spurious or due to procedural differences. Category membership is performed as efficiently by the right hemisphere as by the left. The finding that RTs favored the visual field ipsilateral to the responding hand in Group D-G provides additional evidence in this regard (Moscovitch, 1973). In contrast, category matching apparently relies upon mechanisms unique to the left hemisphere. Their involvement, however, does not appear immediately, as shown by the finding that the RVF advantage in this task occurred only when it followed category membership (i.e., in Group D-G). Consequently, other variables besides the nature of the task itself must be selectively influencing the strategies by which category matching is performed. Two obvious variables are the amount of laterality training and familiarity with the three-letter word list used in both tasks. Word familiarity has been previously implicated as a potent variable in laterality experiments (Hardyck, Tzeng, \& Wang, 1978), so the second experiment was designed to determine its effect, if any, on the emergence of the RVF advantage in Gross's category matching task.

\section{EXPERIMENT 2}

\section{Method}

Subjects. Twenty subjects (14 males, 6 females) from the staff and student populations of the departments of Geology, Oceanography, and Psychology served in this experiment. None had participated in Experiment 1. All the subjects were right-handed, with laterality quotients on the Edinburgh Inventory (Oldfield, 1971) ranging from $.74-10$ and no reported incidence of familial sinistrality among parents or siblings. A high level of performance

Table 2

Petcentages of False Positives (FP) and False Negatives (FN) in Experiment 1

\begin{tabular}{|c|c|c|c|c|c|c|c|c|c|c|c|c|c|c|c|c|}
\hline \multirow[b]{4}{*}{ Hand } & \multicolumn{8}{|c|}{ Group D-G } & \multicolumn{8}{|c|}{ Group G-D } \\
\hline & \multicolumn{4}{|c|}{ Membership (D) } & \multicolumn{4}{|c|}{ Matching (G) } & \multicolumn{4}{|c|}{ Matching (G) } & \multicolumn{4}{|c|}{ Membership (D) } \\
\hline & \multicolumn{2}{|c|}{ FP } & \multicolumn{2}{|c|}{ FN } & \multicolumn{2}{|c|}{$\mathrm{FP}$} & \multicolumn{2}{|c|}{ FN } & \multicolumn{2}{|c|}{$\mathbf{F P}$} & \multicolumn{2}{|c|}{ FN } & \multicolumn{2}{|c|}{ FP } & \multicolumn{2}{|c|}{ FN } \\
\hline & LVF & RVF & LVF & RVF & LVF & RVF & LVF & RVF & LVF & RVF & LVF & RVF & LVF & RVF & LVF & RVF \\
\hline $\begin{array}{l}\text { Left } \\
\text { Right }\end{array}$ & $\begin{array}{r}8.7 \\
13.7\end{array}$ & $\begin{array}{l}15.0 \\
11.2\end{array}$ & $\begin{array}{l}6.2 \\
8.7\end{array}$ & $\begin{array}{l}8.7 \\
5.0\end{array}$ & $\begin{array}{l}6.2 \\
3.7\end{array}$ & $\begin{array}{l}6.2 \\
2.5\end{array}$ & $\begin{array}{r}1.2 \\
10.0\end{array}$ & $\begin{array}{l}1.2 \\
2.5\end{array}$ & $\begin{array}{l}1.2 \\
8.7\end{array}$ & $\begin{array}{r}7.5 \\
13.7\end{array}$ & $\begin{array}{l}8.7 \\
8.7\end{array}$ & $\begin{array}{l}7.5 \\
7.5\end{array}$ & $\begin{array}{l}10.0 \\
10.0\end{array}$ & $\begin{array}{l}8.7 \\
8.7\end{array}$ & $\begin{array}{l}3.7 \\
6.2\end{array}$ & $\begin{array}{l}1.2 \\
2.5\end{array}$ \\
\hline Average & 11.2 & 13.1 & 7.5 & 6.8 & 5.0 & 4.4 & 5.6 & 1.9 & 5.0 & 10.6 & 8.7 & 7.5 & 10.0 & 8.7 & 5.0 & 1.9 \\
\hline
\end{tabular}

Note-Errors are shown by task, visual field, and responding hand for both groups. 
accuracy, as in Experiment 1, was also required for inclusion in the study. The subjects were divided equally into two groups (see Procedure); mean laterality quotients for these groups were .90 (Group C) and 87 (Group T).

Stimuli. Two lists of words denoting animals and two lists of words denoting parts of the human body were the verbal stimuli for this experiment. Each list contained five three-letter words: Animal List 1 (cow, dog, hen, pig, rat), Animal List 2 (ant, cat, eel, fox, owl), Body List 1 (eye, hip, jaw, leg, rib), and Body List 2 (arm, ear, gut, lip, toe). The words making up the two animal lists and two body lists were roughly equated for word frequency using the norms provided by Kučera and Francis (1967).

Two sets of 64 stimulus configurations were constructed from the combination of corresponding word lists (i.e., Animal and Body Lists 1, and Animal and Body Lists 2). Individual configurations consisted of two words oriented vertically, one word positioned for foveal presentation and one for lateral presentation. Each set contained 32 "same" units and 32 "different" units. "Same" units were pairs of words belonging to the same category (i.e., both animals or both body parts), with equal numbers of units from each list. "Different" units were pairs of words belonging to different categories (i.e., one animal word and one body word). Each "same" and "different" unit had two configurations, one with the lateral word to the left of the foveal word and one with the lateral word to the right of the foveal word.

Pairs of words were typed on high-reflectance Chrome-Kote paper with a 12-pitch uppercase Adjutant typeface. ${ }^{1}$ The stimulus pairs appeared black on white backgrounds when photographed and mounted in $35-\mathrm{mm}$ glass slides. When viewed at a distance of $58 \mathrm{~cm}$, the words subtended visual angles of $7.4 \mathrm{deg}$ vertical and $1.0 \mathrm{deg}$ horizontal. The distance between the point of fixation (center of the foveal word) and the inner edge of the lateral word subtended a visual angle of $6.6 \mathrm{deg}$.

Apparatus. The apparatus was the same as that used in Experiment 1.

Procedure. The instructions for this experiment were identical to the instructions for Task $G$ in Experiment 1 . In brief, the subjects were told to press the response button only on trials in which the lateral and foveal words belonged to the same category. A total of 128 trials, divided into two 64-trial blocks, were run. Each block contained equal numbers of positive ("same") and negative ("different") trials. A short rest period intervened between blocks. For the control group, the set of 64 stimulus configurations was identical for both blocks of trials, although the visual field location of the lateral word in each configuration was counterbalanced between blocks, and a different trial order was used. For the transfer group, a different set of configurations was used in each 64-trial block. Half of the subjects in each group used their right index finger for responding, and half used their left. Prior to the first block of trials, the subjects from both groups were given 12 practice trials, using four-letter animal and body words (see Procedure of Experiment 1). At the end of the experiment, a surprise recall test was given to each subject. The subjects were asked to write on a sheet of paper all of the words that they remembered from the second block of 64 trials. Three minutes were allotted for the test.

Data analysis. The selection of data and method of analysis were the same as those used in Experiment 1.

\section{Results and Discussion}

The mean RTs for correct responses as a function of trial block, visual field, and responding hand are shown for both groups in Table 3. Once again, responding hand had no particular effect on performance, so means collapsed across this variable are also presented.

The category matching data clearly parallel the corresponding first block/second block data obtained in Experiment 1. There appear to be no visual field differences in RTs during the first block of 64 trials but an appreciable RVF advantage during the second block.

Post hoc statistical analyses of each group's data lead to the same conclusion as does a casual perusal of the sample data. For the control group, LVF and RVF RTs did not differ during first-block category matching $[F(3,27)=.03]$. RVF RTs during secondblock matching were substantially shorter, however, than the LVF and RVF RTs from the first block $[F(3,27)=5.56]$, while second-block LVF RTs fell between these two extremes $[F(3,27)=.10]$. Clearly, our statistical decisions imply $L_{V F}=R_{1} F_{1}>L V_{2}>$ $R F_{2}$. Stated otherwise, no visual field differences occurred during the first block, but a RVF advantage appeared during the second block. RTs for both fields were also shorter during second- than during first-block matching. Of the 10 control subjects, eight showed a RVF advantage in RTs during the second block of trials compared with four during the first block.

For the transfer group, RTs during the first block also did not differ as a function of visual field $[F(3,27)$ $=.00$ ]. During the second block of trials, however, this group also showed a RVF advantage $[F(3,27)=$ 2.26]. When second-block RTs averaged across visual fields are compared with the corresponding firstblock RTs, no difference is found $[F(3,27)=.03]$. What this analysis implies about the transfer group is: $\mathbf{L V F}_{2}>\mathbf{L V F}_{1}=\mathbf{R V F}_{1}>\mathbf{R V F}_{2}$. Consequently, our conclusions concerning within-block visual field effects are the same as they were for the control group. Eight of the 10 transfer subjects showed secondblock RVF advantage, but only six did during the first block.

The error data for this experiment, shown in Table 4, gave no indication of speed-accuracy tradeoffs. False

Table 3

Mean Reaction Times (in Milliseconds) for Correct Responses in Experiment 2

\begin{tabular}{|c|c|c|c|c|c|c|c|c|}
\hline \multirow[b]{3}{*}{ Hand } & \multicolumn{4}{|c|}{ Control Group } & \multicolumn{4}{|c|}{ Transfer Group } \\
\hline & \multicolumn{2}{|c|}{ First Block } & \multicolumn{2}{|c|}{ Second Block } & \multicolumn{2}{|c|}{ First Block } & \multicolumn{2}{|c|}{ Second Block } \\
\hline & LVF & RVF & LVF & RVF & LVF & RVF & LVF & RVF \\
\hline $\begin{array}{l}\text { Left } \\
\text { Right }\end{array}$ & $\begin{array}{l}878.0 \\
885.8\end{array}$ & $\begin{array}{l}895.8 \\
883.0\end{array}$ & $\begin{array}{l}837.8 \\
844.6\end{array}$ & $\begin{array}{l}796.2 \\
782.4\end{array}$ & $\begin{array}{l}886.6 \\
889.2\end{array}$ & $\begin{array}{l}899.8 \\
874.2\end{array}$ & $\begin{array}{l}952.0 \\
913.8\end{array}$ & $\begin{array}{l}833.2 \\
877.2\end{array}$ \\
\hline Average & 881.9 & 889.4 & 841.2 & 789.3 & 887.9 & 887.0 & 932.9 & 855.2 \\
\hline
\end{tabular}

Note-Data are shown by task, visual field, and responding hand for both groups. 
Table 4

Percentages of False Positives (FP) and False Negatives (FN) in Experiment 1

\begin{tabular}{|c|c|c|c|c|c|c|c|c|c|c|c|c|c|c|c|c|}
\hline \multirow[b]{4}{*}{ Hand } & \multicolumn{8}{|c|}{ Control Group } & \multicolumn{8}{|c|}{ Transfer Group } \\
\hline & \multicolumn{4}{|c|}{ First Block } & \multicolumn{4}{|c|}{ Second Block } & \multicolumn{4}{|c|}{ First Block } & \multicolumn{4}{|c|}{ Second Block } \\
\hline & \multicolumn{2}{|c|}{ FP } & \multicolumn{2}{|c|}{ FN } & \multicolumn{2}{|c|}{ FP } & \multicolumn{2}{|c|}{ FN } & \multicolumn{2}{|c|}{ FP } & \multicolumn{2}{|c|}{ FN } & \multicolumn{2}{|c|}{ FP } & \multicolumn{2}{|c|}{ FN } \\
\hline & LVF & RVF & LVF & RVF & LVF & RVF & LVF & RVF & LVF & RVF & LVF & RVF & LVF & RVF & LVF & RVF \\
\hline $\begin{array}{l}\text { Left } \\
\text { Right }\end{array}$ & $\begin{array}{l}2.5 \\
7.5\end{array}$ & $\begin{array}{r}5.0 \\
11.2\end{array}$ & $\begin{array}{l}8.7 \\
7.5\end{array}$ & $\begin{array}{l}7.5 \\
7.5\end{array}$ & $\begin{array}{l}7.5 \\
5.0\end{array}$ & $\begin{array}{r}2.5 \\
11.2\end{array}$ & $\begin{array}{l}6.2 \\
6.2\end{array}$ & $\begin{array}{l}5.0 \\
2.5\end{array}$ & $\begin{array}{l}13.7 \\
12.5\end{array}$ & $\begin{array}{l}10.0 \\
13.7\end{array}$ & $\begin{array}{l}8.7 \\
2.5\end{array}$ & $\begin{array}{r}10.0 \\
2.5\end{array}$ & $\begin{array}{r}6.2 \\
10.0\end{array}$ & $\begin{array}{l}6.2 \\
8.7\end{array}$ & $\begin{array}{r}10.0 \\
1.2\end{array}$ & $\begin{array}{l}2.5 \\
2.5\end{array}$ \\
\hline Average & 5.0 & 8.1 & 8.1 & 7.5 & 6.2 & 6.8 & 6.2 & 3.7 & 13.1 & 11.8 & 5.6 & 6.2 & 8.1 & 7.5 & 5.6 & 2.5 \\
\hline
\end{tabular}

Note-Errors are shown by task, visual field, and responding hand for both groups.

positives, averaged over responding hand, did not differ by visual field for either group during either block of trials [control group: $F(3,27)=.56$ and .02 for first and second block, respectively; transfer group: $F(3,27)$ $=.05$ and .01 for first and second block, respectively]. A similar absence of visual field effect was found for false negatives [control group: $F(3,27)=.04$ and .60 for first and second block, respectively; transfer group: $F(3,27)=.02$ and .63 , respectively].

Considering both the RT and error results, we reanalyzed the RT data from both groups as if they came from a single group. (We believe that the between-groups differences in the absolute values of the second-block RTs are simply due to sampling error.) Table 5 shows the RTs averaged across groups. Post hoc analyses of these data show that (1) there were no visual field differences during the first block $[F(3,57)=.01],(2)$ LVF RTs during the second block did not differ from first-block $R T s[F(3,57)=.00]$, and (3) RVF RTs during the second block were appreciably shorter than the three other RTs $[F(3,57)$ $=4.70]$. Taken together, these decisions imply that the ordering of RTs for this experiment was as follows: $\mathrm{LVF}_{1}=\mathrm{RVF}_{1}=\mathrm{LVF}_{2}>\mathrm{RVF}_{2}$.

On the memory test, the control subjects recalled an average of 7.9 words, and transfer subjects recalled an average of 8.0 words of a possible 10 . The average number of intrusions was 0.0 and 1.1 , respectively, for each group. No between-groups differences were evident for recall $[F(1,18)=.04]$, but more intrusions occurred in the transfer group $[F(1,18)$ $=11.25]$. The latter finding is not surprising since there was no additional list to cause intrusions for the control group. The recall result, however, is im-

Table 5

Mean Reaction Times (in Milliseconds) for Correct Responses in Experiment 2

\begin{tabular}{ccccc}
\hline \multicolumn{2}{c}{ First Block } & & \multicolumn{2}{c}{ Second Block } \\
\cline { 5 - 5 } LVF & RVF & & LVF & RVF \\
\hline 884.9 & $\mathbf{8 8 8 . 2}$ & & $\mathbf{8 8 7 . 0}$ & 822.3 \\
\hline
\end{tabular}

Note-Data have been averaged across responding hands and across groups. portant because it indicates that word familiarity for the common (second) list was equal across groups. We had hoped for a somewhat different result (i.e., less word familiarity in the transfer than in the control group).

Nonetheless, the RT data from this experiment confirm the findings obtained in Experiment 1. Visual field differences in RTs on Gross's (1972) category matching task do not appear immediately. Their emergence could conceivably result from a general practice effect, although word familiarity has not yet been ruled out. A practice effect seems unlikely, however, because when both groups are considered together, the only between-block effect was a decrease in RVF RTs. Practice would most likely cause a decrease in RTs across both visual fields.

\section{GENERAL DISCUSSION}

The major finding of this study is that the laterality results of both Day (1977, Experiment 3) and Gross (1972, Experiment 1) can be replicated with all procedural variables held constant. Using concrete words as stimuli, we found no visual field differences in RTs on Day's category membership task, but faster RVF than LVF RTs on Gross's category matching task.

Our results also showed, however, that the RVF advantage on Gross's task does not appear immediately. Subjects in each experiment failed to show any visual field differences in category matching RTs within the first block of 64 trials. We cannot determine if similar between-blocks effects were obtained by Gross (1972, Experiment 1) because she did not report her data by blocks. Nonetheless, it is interesting to note that we observed a RVF advantage on the category matching task whether the first and second blocks employed (1) the same words and task, (2) different words and the same task, or (3) the same words and different tasks.

We prefer to interpret the absence of visual-field differences in category membership and in first-block category matching as an indication that the right hemisphere in normal right-handers can perform 
these tasks as efficiently as the left hemisphere under our procedural conditions. Two alternative interpretations of our data, however, should be addressed.

The first alternative argues that subjects with relatively low laterality quotients contributed disproportionately to the absence of visual field differences because they were less "right-handed" and, hence, less lateralized in their hemispheric abilities. This argument predicts that subjects with low quotients would more likely produce LVF advantages (or relatively small RVF advantages) than subjects with higher quotients. Stated otherwise, LVF minus RVF reaction time differences should be positively correlated with degree of handedness. Our observed correlations were .22 for category membership and .02 for first-block category matching. Clearly, our results cannot be accounted for in any straightforward way by heterogeneity in our sample of normal right-handers.

The second alternative concerns the inclusion of female subjects in our experiment. From most indications, females tend to be less lateralized in language functioning than males (e.g., Bradshaw, Gates, \& Nettleton, 1977; Levy \& Reid, 1978; but see Piazza, 1980). Unfortunately, we cannot analyze, for statistical reasons, a Visual Field by Sex effect since there were unequal numbers of males and females in the various experimental groups. We did, however, carefully examine the mean visual field RTs for each sex in order to determine whether or not there were any obvious departures from the trends in the average data. The only possible indication of sex differences came from Group G-D in Experiment 1. All three female subjects in that group showed a LVF advantage in RTs during first-block category matching, while only one of seven males showed a similar advantage. This might indicate that the absence of visual field differences in this group during category matching was spurious. We did, however, replicate our category matching results in Experiment 2, in which there were no obvious sex differences. Furthermore, two other observations would seem to argue against a simple interpretation on the basis of sex. First, the eventual emergence of the RVF advantage in category matching would remain to be explained. Second, both Day (1977) and Gross (1972) included female subjects in their experiment, so their differential laterality effects would be difficult to reconcile on this account. All things considered, we believe that these differential effects and our replication of them reflect true task differences.

The strategy for category matching and how it differs from category membership strategies is, however, still unclear. We entertained the hypothesis that word familiarity was somehow related to the emergence of a RVF advantage in category matching, but our test of this hypothesis failed to produce a betweengroups difference in familiarity. Hardyck, Tzeng, and Wang $(1977,1978)$ previously reported that the usual visual field difference in $R T$ s with verbal and spatial stimuli do not appear if new stimuli are used on every trial. Differences in the predicted directions do appear, however, if stimuli are frequently repeated during a task. Hardyck et al. claim that these results indicate that cerebral lateralization effects may not reflect hemispheric specialization for immediate reaction to particular types of stimuli but, rather, a specialization for the memory of these stimuli. The implications for word familiarity effects are obvious.

Whether the memory distinction proposed by Hardyck et al. $(1977,1978)$ will substantially change our present conceptualizations of hemispheric differences remains to be seen. We should point out, however, that the category matching data from Experiment 2 suggest that the memory interpretation may not be entirely accurate. We found a distinct RVF advantage in category matching RTs during the second block of training trials, whether or not the word list was changed between blocks. Furthermore, individual foveal/lateral word pairs appeared only once within each block, so a stimulus repetition effect (Hardyck et al., 1977, 1978) was precluded. One could argue, however, that our data do not address the issue raised by Hardyck et al. because recall performance (the index of word familiarity) was equally strong in both the transfer and control groups. Nonetheless, if recall of second-block words was so good even with a change in lists, then recall of first-block words after first-block training should be just as good, and visual field differences were absent initially. The resolution of this issue awaits further research.

We should also point out that the design of the Hardyck et al. $(1977,1978)$ research contains a potential confound. In those experiments in which the authors found laterality effects, subjects received at least twice as many trials as in experiments in which no effects were found. Consequently, a simple trial (practice) effect might be invoked to explain their results. A series of experiments by Ward and Ross (1977) provides some support for the notion that laterality effects are more likely to be influenced by the amount of "practice" on a task than by transfer (or lack of transfer) from one stimulus set to another. They found a RVF advantage for identifying laterally presented letters in a backward masking task, but the asymmetry vanished after the first session. This pattern was obtained regardless of whether the stimulus set remained the same or was changed between sessions.

Interestingly, the eventual disappearance of visual field differences in the Ward and Ross (1977) study stands in direct contrast to the delayed emergence of laterality effects observed in the present study and the Hardyck et al. $(1977,1978)$ experiments. Other investigators have also reported attenuation or elim- 
ination of perceptual asymmetry with extended training on various laterality tasks (Hellige, 1976; Hellige, Cox, \& Litvac, 1979; Kallman \& Corballis, 1975). Reasons for these differences are not entirely clear. However, one possibility is that shifts in hemispheric dominance depend on the relative capacities of the two hemispheres to improve with training and that these capacities vary with the nature of the specialized processing demands associated with a given task. It is also conceivable that perceptual asymmetries are subject to "fatigue" effects (Kallman \& Corballis, 1975) as well as to "practice" effects.

Our first experiment showed that category membership not only produced different laterality effects than did category matching, but also that it was generally performed faster than category matching. Although this latter effect can be seen in the original results of Day (1977, Experiment 3) and Gross (1972, Experiment 1), numerous procedural differences between these two studies preclude direct comparisons. The difference in speed observed here, however, with procedural differences held constant, provides further evidence that subjects use different strategies for performing each task. ${ }^{2}$ In the matching task, subjects must determine the superordinate categories for $t w o$ words and match them, while in the membership task, they must determine only one category for comparison with the foveal category word.

Previous research has shown that different visual field asymmetries can be produced in different tasks even when the stimuli used are identical (e.g., Jonides, 1979; Levy \& Trevarthen, 1976). The present study shows very similar tasks can also produce different patterns of perceptual asymmetries. It seems a bit premature to speculate on the theoretical import of our findings, since we have yet to determine exactly what variables contribute to the differential effects observed here. Clearly, we can safely say that hemispheric differences are not bound in any simple way by the stimuli or the tasks that subjects encounter. We would like to offer, however, this tentative interpretation of our results. Suppose that superordinate category information can be determined with equal rapidity by the left and right hemispheres, as suggested by our category membership data and those of Day (1977). Consider, now, the possibility that after sufficient practice or repeated activation, the left hemisphere can handle simultaneous lexical decision making, while the right hemisphere cannot. Since the category matching task requires the determination of two superordinate categories, this capacity could produce a left hemisphere (RVF) advantage. In the category membership task, only one superordinate need be determined, since the other is given by the foveal stimulus. Therefore, no visual field difference is observed with this task. While this post hoc explanation is consistent with our results, further research will be necessary to determine its accuracy.

\section{REFERENCES}

Bradshaw, J. L., Gates, A., \& Netti.titon, N. C. Bihemispheric involvement in lexical decisions: Handedness and a possible sex difference. Neuropsychologia, 1977, 15, 277-286.

Bryden, M. P., \& AlitanD, F. Visual hemifield differences depend on typeface. Brain and Language, 1976, 3, 191-200.

DAY, J. Right-hemisphere language processing in normal righthanders. Journal of Experimental Psychology: Human Perception and Performance, 1977, 3, 518-528.

Gazzanigia, M. S. The bisected brain. New York: AppletonCentury-Crofts, 1970.

Gross, M. M. Hemispheric specialization for processing of visually presented verbal and spatial information. Perception \& Psycho. physics, 1972, 12, 357-363.

Hahdyck, C., Tzenci, O. J. L.. \& Wanc;, W. S.-Y. Cerebral lateralization effects in visual half-field experiments. Nature, 1977, 269, 705-707.

Hardyck, C., Tzenc;, O. J. L., \& Wanc, W. S.-Y. Cerebral lateralization of function and bilingual decision processes: Is thinking lateralized? Brain and Language, 1978, 5, 56-71.

HecaEN, H. Right hemisphere contribution to language function. In P. A. Buser \& A. Rougeul-Buser (Eds.), Cerebral correlates of conscious experience (INSERM Symposium No. 6). Amsterdam: Elsevier, 1978.

Held.Jik, J. B. Changes in same-different laterality patterns as a function of practice and stimulus quality. Perception \& Psychophysics, 1976, 20, 267-273.

Hellige, J. B., Cox, P. J., \& Litvac, L. Information processing in the cerebral hemispheres: Selective hemispheric activation and capacity limitations. Journal of Experimental Psychology: General, 1979, 108, 251-279.

Jonides, J. Left and right visual field superiority for letter classification. Quarterly Journal of Experimental Psychology, 1979, 31, 423-439.

Kalliman, H. J., \& Corbalids, M. C. Ear asymmetry in reaction time to musical sounds. Perception \& Psychophysics, 1975, 17, 358-370.

KUĆERA, H., \& FRANCIS, W. N. Computational analysis of presentday American English. Providence, R.I: Brown University Press, 1967.

Levy, J., \& Rkin, M. Variations in cerebral organization as a function of handedness, hand posture in writing, and sex. Journal of Experimental Psychology: General, 1978, 107. 119-144.

Levy, J., \& Trevartuen, C. Metacontrol of hemispheric function in human split-brain patients. Journal of Experimental Psychology: Human Perception and Performance, 1976, 2, 299-312.

McFarland, K., McFarland, M. L., Bain, J. D., \& Asit ton, R. Ear differences of abstract and concrete word recognition. Neuropsychologia, 1978, 16, 555-561.

Moscovitch, M. Language and the cerebral hemispheres: Reaction time studies and their implications for models of cerebral dominance. In P. Pliner, L. Krames, \& T. Alloway (Eds.), Communication and affect: Language and thought. New York: Academic Press, 1973.

OldFifi.D, R. C. The assessment and analysis of handedness: The Edinburgh Inventory. Neuropsychologia, 1971, 9, 97-113.

Piazza, D. M. The influence of sex and handedness in the hemispheric specialization of verbal and nonverbal tasks. Neuropsychologia, 1980, 18, 163-176.

RODGER, R. S. The number of non-zero, post-hoc contrasts from ANOVA and error rate. I. British Journal of Mathematical and Statistical Psychology, 1975, 28, 71-78. (a)

RODGER, R. S. Setting rejection rate for contrasts selected post-hoc when some nulls are false. British Journal of Mathematical and Statistical Psychology, 1975, 28, 214-232. (b)

SCHErrÉ, H. A method of judging all contrasts in the analysis of variance. Biometrika, 1953, 40, 87-104.

Stantsiman, A. A review of right hemisphere linguistic capabilities. Psychological Bulletin, 1977, 84, 503-528.

WAHD, T. B., \& Ross, L. E. Laterality differences and practice 
effects under central backward masking conditions. Memory \& Cognition, 1977, 5, 221-226.

ZaIDEL, A. Lexical organization in the right hemisphere. In P. A. Buser \& A. Rougeul-Buser (Eds.), Cerebral correlates of conscious experience (INSERM Symposium No. 6). Amsterdam: Elsevier, 1978.

\section{NOTES}

1. The change in type set was inadvertent. The Adjutant font does have a print-like quality, however, much like the Orator font used in Experiment 1. Consequently, our results should not be differentially affected (Bryden \& Allard, 1976).

2. For example, since there were only two category words in the category membership task, and since their word lengths were quite different, subjects could discriminate between the category words without actually reading them. In fact, some of our subjects reported that they identified which category word was present on any given trial by its length or its middle two letters. This particular strategy may account for all or part of the task differences in RT, although it cannot account for the differential visual field effects. In Day's original experiment, there were six different categories, and one would suppose that reading them was required to perform the task. Yet, Day found no visual field difference with concrete categories and a RVF advantage with abstract categories.

(Received for publication September 2, 1980; accepted February 18, 1981.) 